\title{
Methodology of a Troposphere Effect Mitigation Processor for SAR Interferometry
}

\author{
Nico Adam http://orcid.org/0000-0002-6053-0105
}

\begin{abstract}
Troposphere effect mitigation based on numerical weather prediction (NWP) is an actual research topic in SAR interferometry (InSAR) and especially in persistent scatterer interferometry (PSI). This is the reason, a scientific troposphere effect mitigation processing system has been developed. The objective of this paper is to provide the methodology of four developed algorithms, demonstrate application examples, discuss the methods characteristic and recommend techniques for operational systems.
\end{abstract}

Index Terms-persistent scatterer interferometry (PSI), atmospheric phase screen (APS), numerical weather prediction (NWP), wide area processing (WAP)

\section{INTRODUCTION}

$\mathrm{R}$ adar wave propagation effects being the most dominant error source in InSAR data has long been recognized [1], [2],[3],[4],[5],[6]. The three main effects relevant for InSAR i.e. (a) hydrostatic delays, (b) wet delays, and (c) ionosphere effects are first considered by Zebker et al. [4]. Later on, Hanssen [5], [7] has completed this list with (d) the liquid water effect. The ionosphere effects (c) are mitigated straight forward by the split-spectrum method [8], [9] based on the fact it is a dispersive medium [10], [3]. In recent years, the mitigation of troposphere impacts (a) and (b) have attracted strong interest, however their mitigation is still a challenge. Williams et al. [6] have analyzed the spatial and temporal characteristic of InSAR tropospheric effects by the spatial and temporal structure function and provided the covariance between two points separated in time and/or space. Much progress has been achieved, by optimizing the algorithmic InSAR processing scheme. Essentially, Zebker et al. [4] have suggested the central principle for mitigation of troposphere

N. Adam, "Methodology of a Troposphere Effect Mitigation Processor for SAR Interferometry," in IEEE Journal of Selected Topics in Applied Earth Observations and Remote Sensing, vol. 12, no. 12, pp. 5334-5344, Dec. 2019. doi: 10.1109/JSTARS.2019.2957919, online http://ieeexplore.ieee.org/stamp/stamp.jsp?tp=\&arnumber=8945244\&isnumbe $\mathrm{r}=8979194$ effects for surface deformation monitoring. I.e. knowing the troposphere is uncorrelated in time, averaging of $N$ deformation measurements by stacking mitigates the effects standard deviation by a factor $\sqrt{N}$. This principle has been brought to perfection by Ferretti et al. [11], [12], [13] via the long time span series analysis technique named persistent scatterer interferometry (PSI). Another well-known stacking development is the Small BAseline Subset (SBAS) algorithm [14]. Although stacking techniques now separate the InSAR measurement components i.e. topography, deformation, and troposphere, mitigating the tropospheric contribution is still vital. Due to the relation between the number of scenes and the expected variance of errors of the atmospheric estimates provided by Emardson et al. [15] and Rocca [16], compensation of troposphere by independent data reduces the number of required radar scenes or improves the deformation measurement precision [6], [17]. This is a direct consequence of the principle that the correction of the atmosphere and the stacking are complementary as pointed out by Williams et al. [6]. The correction of the atmosphere effects by independent data is denoted also calibration [6], [18], [15], [19]. In order to implement this, many different tropospheric mitigation techniques have been developed that reduce (a) hydrostatic delays and/or (b) wet delays because of their different characteristics. Hydrostatic delays result in a vertically stratified component, which correlates with the topography. For constant altitudes, it is spatially smooth because it can be modelled by temperature and pressure of the local troposphere. The wet delay corresponds to the turbulent water vapor component and is for this reason temporally and spatially very variable and consequently most difficult to compensate. The published calibration techniques can be classified into six categories. A) Vertical stratification directly estimated from InSAR data [20], [21], [22], [23], [24], [25], [26], [27]. (B) GNSS data i.e. based on Zenith Tropospheric (or Total) Delay (ZTD) respectively Zenith Path Delay (ZPD) or Zenith Wet Delay (ZWD) data [28], [6], [18], [15], [19], [29], [30], [31]. (C) Troposphere models using ground meteorological data e.g. Hopfield or Saastamoinen together with a mapping function e.g. Niell [4], [18], [32]. (D) Global coarse resolution meteorological data e.g. NARR, NH3D, ERA-Interim or the Climate Forecast System Reanalysis (CFSR) [33] [34], [35], [25], [36], [37]. (E) Imaging spectrometer e.g. MERIS and MODIS [32], [38], [25], [37] and (F) High resolution weather model data based on numerical weather prediction (NWP) [39], [40], [41], [42], 
IEEE Journal of Selected Topics in Applied Earth Observations and Remote Sensing

[25]. All the above techniques, except the first, use independent auxiliary data.

Extensive research is still being done for troposphere mitigation e.g. in 2017 with the Toolbox for Reducing Atmospheric InSAR Noise (TRAIN) [25] and in 2018 with GACOS [31], [43] or [26], [27]. In the broader context of geosynchronous synthetic aperture radar, troposphere and APS models are also proposed [44], [45], [46]. However, little is published recently on troposphere mitigation based on F) high resolution weather model data based on NWP. Besides, prior research concluded NWP data cannot mitigate the turbulent water vapor [47] and support InSAR simply on long spatial wavelength i.e. longer than $20 \mathrm{~km}$ [48].

This paper's contribution is summarized as follows. First, the presented research demonstrates by practical applications that troposphere mitigation based on NWP is attractive. Particularly, it overcomes limitations of the alternative techniques e.g. the sparse sampling of GNSS (B) and ground meteorological (C) data, the coarse resolution of global meteorological (D) and imaging spectrometer (E) data as well as the acquisition restrictions of imaging spectrometer (cloud free, at day time). Second, the manuscript verifies that NWP techniques (F) are suitable for an operational mitigation service which is relevant for completed (e.g. ERS-1/2, RADARSAT-1), current (e.g. Sentinel-1, TerraSAR-X) and proposed (e.g. Tandem-L) SAR missions. This is a presentday topic as monitoring the Earth's ground motion with millimeter precision from space on a global scale requires operationally troposphere corrections with all SAR scenes as proposed by Eineder et al. [49] or provided by von Allmen et al. [50]. Third, the paper contributes directly to wide area PSI (WAP). In mountainous and non-urban areas, the reference network typically cannot represent the troposphere effect. In this case, atmosphere mitigation is the key to avoid disconnected regions and error propagation. The paper demonstrates the typical SNR improvement of four NWP based methods comparing uncalibrated and troposphere calibrated data for each method independently. Also, it is intended to increase the attention for NWP based troposphere mitigation in the context of WAP. Fourth in the course of the authors developments, four mitigation techniques are implemented and were demonstrated and validated in projects e.g. Terrafirma [24] and a pilot study [51]. Each of these methods has its own characteristic, advantages and limitation. Subject of this paper is to provide the methodology, demonstrate application examples and discuss the methods characteristic.

This paper is organized as follows. Section II describes the methods. Respective application demonstrations are provided in the results section III. In section IV, the characteristics of these methods are discussed. Finally, section $\mathrm{V}$ presents the conclusions.

\section{METHODS}

Numerical weather prediction (NWP) forecasts or hindcasts the atmosphere state based on coarse spatial and temporal initial data through the use of physical modelling and numerical approaches. The Weather Research and Forecasting (WRF) model [52] is an available and well supported implementation of NWP providing the temporal evolution of pressure, temperature and water vapor at high spatial resolution and finally at the time of a SAR acquisition.

The Smith-Weintraub equation forms the basis of the NWP based troposphere effect mitigation [53].

$$
N(\vec{r})=k_{1} \frac{P_{d}(\vec{r})}{T(\vec{r})}+k_{4} \frac{P_{c}(\vec{r})}{T(\vec{r})}+k_{2} \frac{e_{w}(\vec{r})}{T(\vec{r})}+k_{3} \frac{e_{w}(\vec{r})}{T^{2}(\vec{r})}
$$

It models the scaled-up atmospheric refractivity $N(\vec{r})$ [unitless] by the partial pressures of dry air $P_{d}$ and of carbondioxide $P_{c}$, absolute temperature $T$ and the water vapor partial pressure $e_{w}$. Rüger established the scaling constants $k_{1}, k_{2}, k_{3}$ and $k_{4}$ [54]. The range error $d^{N W P}$ can be estimated by the integrated scaled-up refractivity $N(\vec{r})$ along the wave propagation path i.e. the line of sight (LOS)

$$
d^{N W P}=10^{-6} \cdot \int_{R_{\text {scatterer }}}^{R_{\text {sensor }}} N(\vec{r}) d(\vec{r})[\mathrm{m}]
$$

Via the radar wavelength $\lambda$, the atmospheric phase screen (APS) $\varphi^{N W P}$ is calculated by the straight forward relation

$$
\varphi^{N W P}=\frac{4 \pi}{\lambda} d^{N W P}[\mathrm{rad}]
$$

This basic principle is suitable for implementing various methods. CFSR data [55], [56] are common to all and feature the global consistent state of the atmosphere every six hours with ca. $38 \mathrm{~km}$ horizontal resolution starting in year 1979. The data are made available by the National Center for Atmospheric Research (NCAR) and are the input to the NWP producing the right hand side parameters $P_{d / c}, T$ and $e_{w}$ of Eq. (1). In Eq. (2), the integration path can be chosen along the line of sight or in zenith direction. The latter results in a computationally very efficient characterization of the troposphere impact. It can be used for the master selection support which is an indirect mitigation. In contrast, the LOS integration allows a direct calibration and is implemented in the following methods. If one limits to the characterization of the troposphere along vertical profiles, it results the vertical stratification mitigation. A computationally very efficient integration algorithm makes the high resolution APS mitigation and the high resolution and high precision APS mitigation practically feasible. The high precision in the second method is based on the utilization of the PSI data to correct the timing error of the NWP. In the following subsections, the developed methods are described.

\section{A. Master Selection Support}

For PSI on small urban areas, the master selection in the center of the time baseline diagram is a suitable concept [57]. However for the WAP, the master choice is essential for the PSI precision and final quality. Due to the PSI single master concept, the troposphere effect of the master acquisition is 
IEEE Journal of Selected Topics in Applied Earth Observations and Remote Sensing

included in all interferograms. Within PSI, the interferometric APS, i.e. the sum of master and slave effect, is estimated by a reference network. In practice, the PSI processing includes a sparse phase unwrapping followed by a Kriging for the spatial interpolation as well as the separation of noise and the interferometric atmosphere effect. A spatially smooth APS is a requirement for the successful phase unwrapping and Kriging filtering. Conversely, an unsuitable master scene needs to be compensated by a denser reference network. The subject of the master selection support is to predict the tropospheric power for each scene and select a master scene with the spatially least variable APS.

Using the WRF system [52], the atmosphere state is computed at the time of each SAR acquisition with a horizontal resolution of $3 \mathrm{~km} \mathrm{x} 3 \mathrm{~km}$ and 50 vertical levels. Now, the scaled-up atmospheric refractivity $N$ is computed separately for the dry $N_{d r y}$ (i.e. terms with $P_{d}$ and $P_{c}$ ) and the wet $N_{w e t}$ (i.e. terms with $e_{w}$ ) component using Eq. (1) as

$$
N=N_{d r y}+N_{w e t}[\text { unitless }]
$$

Given the WRF variables total pressure $P_{\text {tot }}[\mathrm{hPa}]$, the absolute temperature $T_{K}\left[{ }^{\circ} \mathrm{K}\right]$ and the vapor mixing ratio $Q_{k g / k g}[\mathrm{~kg} / \mathrm{kg}]$, Eq. (4) is composed of

$$
N_{d r y}=k_{1} \frac{0.622 \cdot P_{t o t}}{\left(0.622+Q_{\mathrm{kg} / \mathrm{kg}}\right) \cdot T_{K}}
$$

and

$$
N_{\text {wet }}=\frac{P_{t o t} \cdot Q_{\mathrm{kg} / \mathrm{kg}} \cdot\left(0.622 \cdot k_{3}+k_{2} \cdot T_{K}\right)}{\left(0.622+Q_{\mathrm{kg} / \mathrm{kg}}\right) \cdot T_{K}^{2}}
$$

In doing this, the dry and wet scaled-up atmospheric refractivity is available at a 3D-grid (blue dots in Fig. 1).

For the characterization of the atmosphere, Eq. (2) needs to be implemented. In practice, the zenith direction integration path (Fig. 1) is convenient. A five-point Newton-Cotes integration formula integrates the tabulated data (i.e. the WRF grid) fast and without heavy CPU load. At the end, a metric is needed to compare the expected APS roughness of all scenes. Conventionally, a semivariogram describes this characteristic and is defined by

$$
\gamma(h)=\frac{1}{2 \cdot N_{p}(h)} \sum_{i=1}^{N_{p}(h)}\left\{Z\left(x_{i}\right)-Z\left(x_{i}+h\right)\right\}^{2}\left[\operatorname{rad}^{2}\right]
$$

$N_{p}(h)$ is the number of data pairs $\left\{Z\left(x_{i}\right), Z\left(x_{i}+h\right)\right\}$ which are separated by lag $h$ and $x_{i}$ is the data location. In order to arrive at a straight forward master scene selection, a single value needs to characterize an applicable master scene. It was found that a small mean value of the wet component in the scene area is a suitable criterion. The process flow diagram is visualized in Fig. 2.

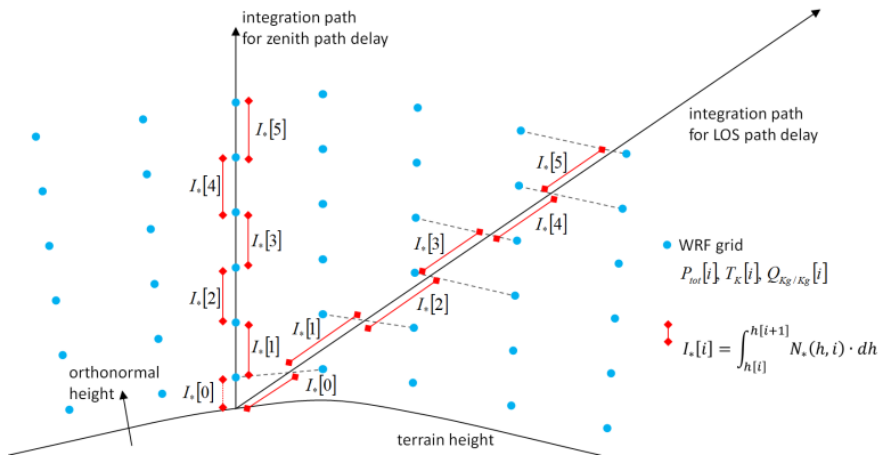

Fig. 1. Integration paths on the NWP data grid.

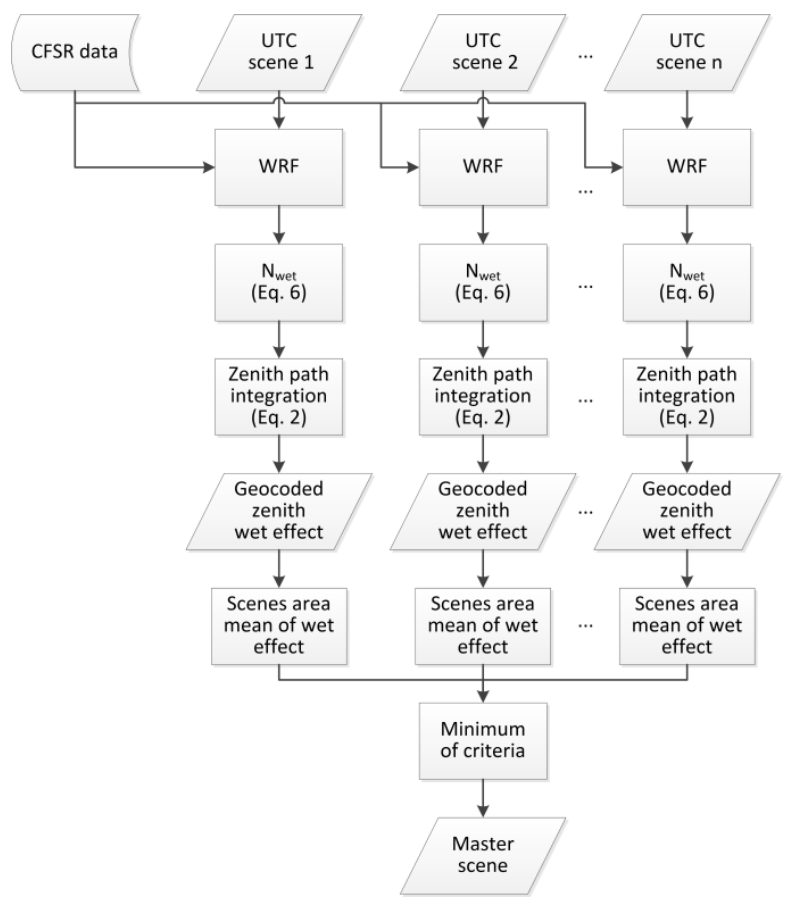

Fig. 2. Process flow diagram of the master selection support.

\section{B. Vertical Stratification Mitigation}

At few scene locations, vertical profiles of the troposphere effect are calculated (Fig. 3). For this method, the integration along the LOS is required and Eq. (2) is implemented by the Gauss-Kronrod quadrature formula. In practice, the increased computation time of this quadrature algorithm is uncritical because a coarsely sampled grid of vertical profiles (e.g. every $10 \mathrm{~km}$ ) is adequate. Starting from points along the vertical profiles (e.g. every $50 \mathrm{~m}$ ), the troposphere range error along the LOS is calculated as a function of altitude for the master and slave atmosphere independently. In Fig. 3, the respective LOS integration path is visualized. Of course, the height dependent interferometric vertical stratification correction is calculated from the difference of these two values (Fig 4 left). Typically, a third order polynomial can approximate this function. In the next step, the coefficients of the fitted polynomials are spatially interpolated into the resolution of the input phase resulting in a unique vertical stratification polynomial for each interferogram sample. Notably, the method is model free i.e. the polynomial approximation makes 
IEEE Journal of Selected Topics in Applied Earth Observations and Remote Sensing

merely the spatial horizontal and vertical interpolation straight forward. Similar to DInSAR topography correction, the DEM is transformed into the master scene SAR geometry. Once the actual height at each interferogram sample is available, it is the input argument for the evaluation of the vertical stratification polynomial which provides the correction phase (Fig. 4 right). The overall process flow diagram is visualized in Fig. 5.

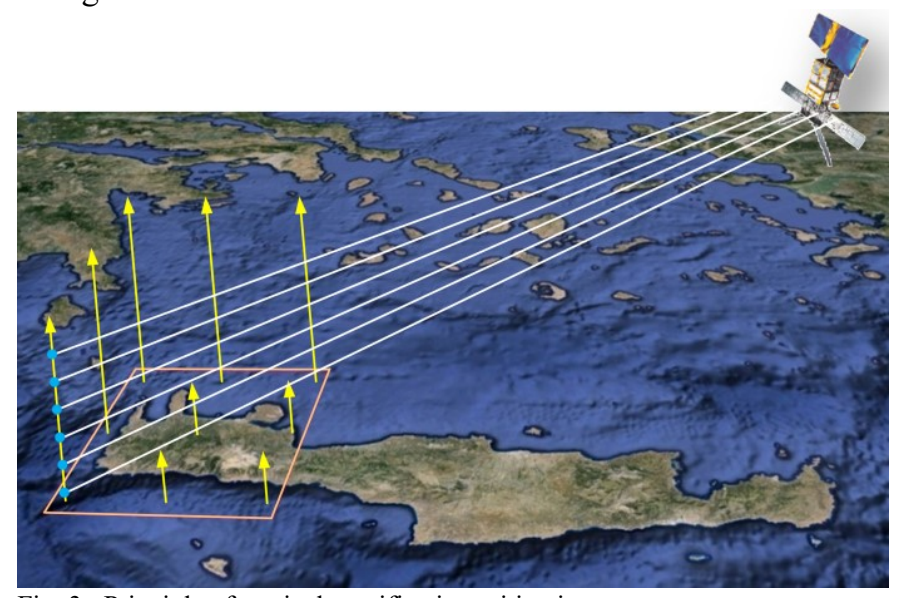

Fig. 3. Principle of vertical stratification mitigation.
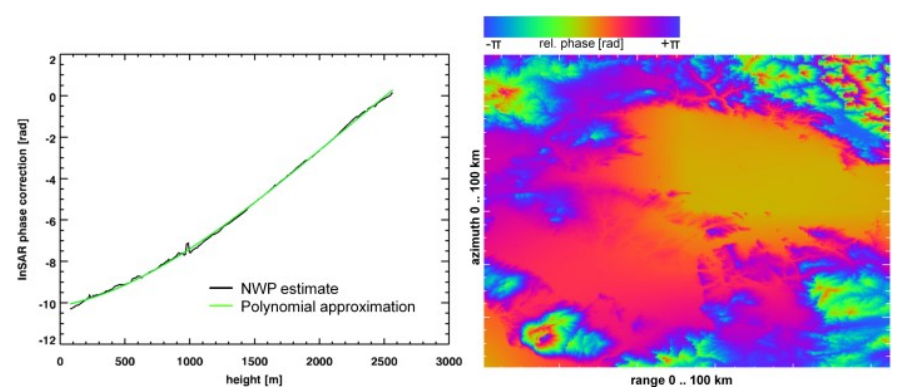

Fig. 4. Interferometric stratification correction. Left: polynomial for a single sample, right: $100 \mathrm{~km} \times 100 \mathrm{~km}$ interferogram correction.

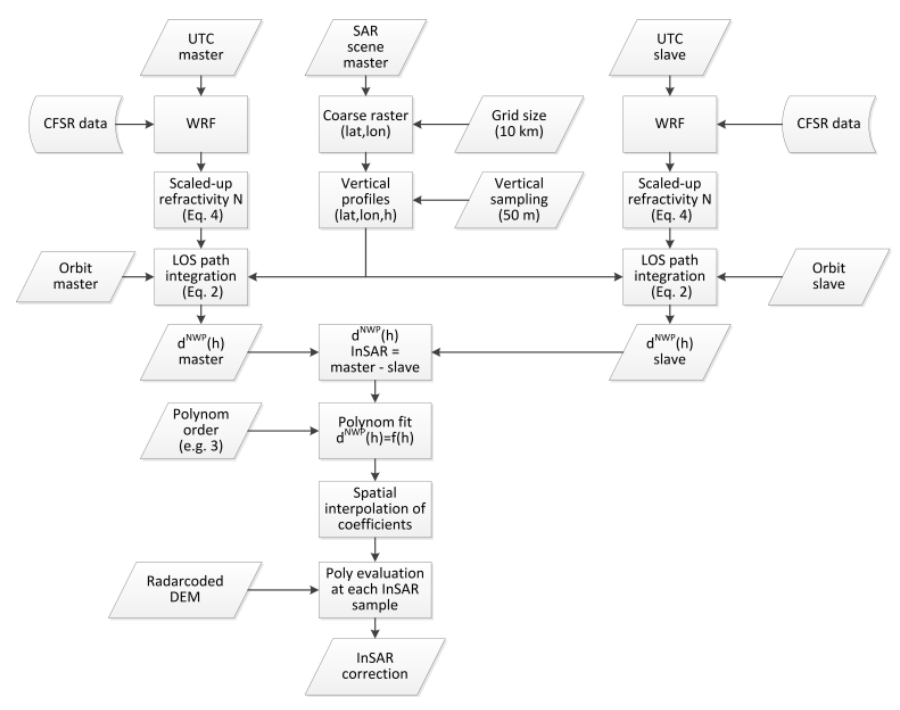

Fig. 5. Process flow diagram of the vertical stratification mitigation.

\section{High Resolution APS Mitigation}

For high resolution mitigation, the integration along the real
LOS needs to be performed on the raster of the master scene directly using the $3 \mathrm{D}$ positions of the resolution cells from a radarcoded DEM as the start locations. Fig. 6 visualizes the principle and that this algorithm copes with local topography as well as the local distribution of humidity, temperature and air pressure. In this figure, the points $\mathrm{P}_{1}$ and $\mathrm{P}_{2}$ provide a typical example of how the APS can change significantly from sample to sample caused by topography and the respective integration distances $L_{P 1}$ and $L_{P 2}$ through a NWP grid cell. A general problem of a straight forward implementation using the adaptive quadrature is the execution time making it practically infeasible. A newly developed algorithm approximates the integral of each LOS segment (red lines in Fig. 1) between vertical WRF grid layers [24].

For temperature $T_{K}$ and water vapor $Q_{k g / k g}$, the segmentwise parametric integration is based on a linear interpolation ( $L i)$ at orthometric height $h\left\{h \mid h_{1} \leq h \leq h_{2}\right\}$ parameterized by the adjacent WRF grid layer heights $h_{1}$ and $h_{2}$ as well as the respective values $v_{1}, v_{2}$ of $T_{K}$ and $Q_{\mathrm{kg} / \mathrm{kg}}$.

$$
L i\left(h, h_{1}, h_{2}, v_{1}, v_{2}\right)=\frac{h \cdot\left(v_{1}-v_{2}\right)-h_{2} \cdot v_{1}+h_{1} \cdot v_{2}}{h_{1}-h_{2}}
$$

The total pressure $P_{\text {tot }}$ is represented along orthometric height by a log-linear interpolation ( $\log \operatorname{LinInt})$

$$
\begin{aligned}
& \log \operatorname{LinInt}\left(h, h_{1}, h_{2}, v_{1}, v_{2}\right) \\
& =\operatorname{Exp}\left(\operatorname{Li}\left(h, h_{1}, h_{2}, \log \left(v_{1}\right), \log \left(v_{2}\right)\right)\right)
\end{aligned}
$$

between the adjacent WRF grid layers at heights $h_{1}$ and $h_{2}$ and their respective values $v_{1}, v_{2}$ of $P_{\text {tot }}$. Inserting this parameterization into Eq. (5) and (6), the interpolated values at orthometric height $h\{h \mid h[i] \leq h \leq h[i+1]\}$ in the vertical WRF grid segment with index $i$ depend only on the neighbouring layer heights $h[i], h[i+1]$ and the respective layer values $T_{K}[i], \quad T_{K}[i+1], \quad Q_{k g / k g}[i], \quad Q_{k g / k g}[i+1]$, $P_{\text {tot }}[i]$ and $P_{t o t}[i+1]$. Fig. 7 visualizes the respective parameters. As a result, the scaled-up refractivity

$$
\begin{gathered}
N(h, i)=f\left(h, T_{K}[i], T_{K}[i+1], Q_{k g / k g}[i], Q_{k g / k g}[i\right. \\
\left.+1], P_{\text {tot }}[i], P_{\text {tot }}[i+1]\right)
\end{gathered}
$$

can now be evaluated directly along the vertical line segment. Unfortunately, the non-linear Eq. (10) cannot be integrated analytically and numerical integration is the straight forward solution. In order to gain performance i.e. evaluating the integral directly, Eq. (10) is approximated by a Taylor series around the center point of the respective interval $[h[i], h[i+$ 1]]. For a typical vertical profile, the maximum relative error of the dry effect refractivity is less than $0.5 \%$ and of the wet effect less than $1.5 \%$ with a first order series expansion. With a second order, the maximum relative error reduces to $0.02 \%$ for the dry effect and to $0.06 \%$ for the wet effect refractivity [24]. For the Taylor series, an analytical integral is evaluated and the LOS incidence angle $\vartheta$ with respect to the orthometric height axis is corrected by the factor $\cos (\vartheta)^{-1}$ [24] (Fig. 7). 
IEEE Journal of Selected Topics in Applied Earth Observations and Remote Sensing

As a consequence, the integral $I[i]$ for each segment is calculated directly from the intersection values of the LOS with the vertical WRF grid layers (orange dots in Fig. 7). An algorithm similar to raytracing provides these intersection points. In doing this, 3D-interpolation and numerous function evaluations can be avoided and the troposphere range effect $d^{N W P}$ is the sum of few segment integral values. The detailed process flow diagram is visualized in Fig. 8.

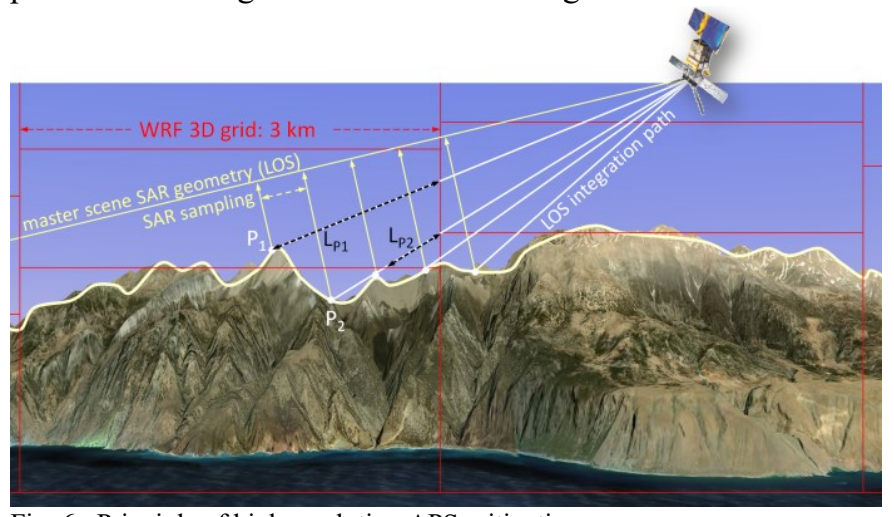

Fig. 6. Principle of high resolution APS mitigation.

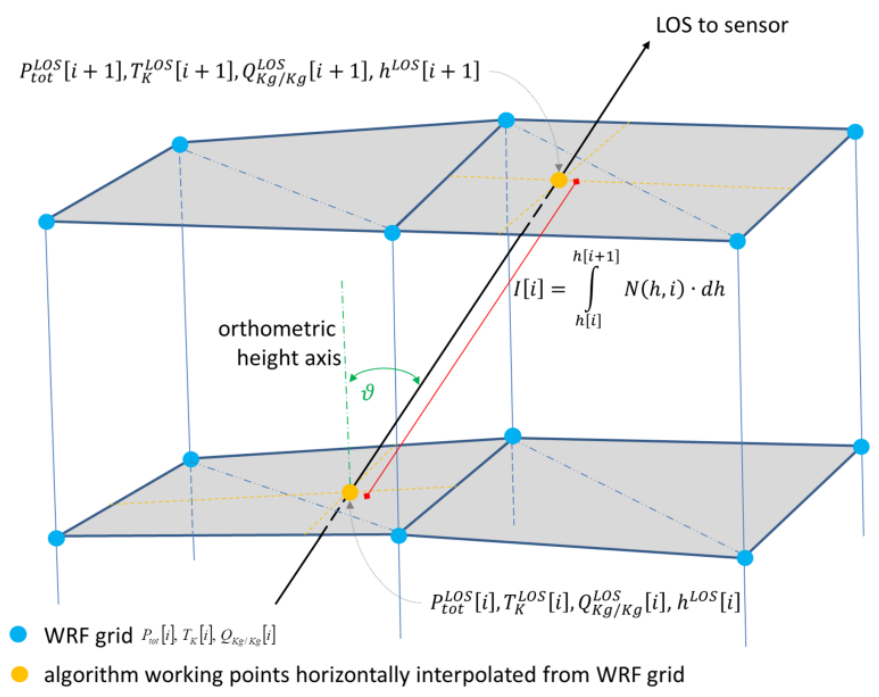

Fig. 7. Geometry and principle of the segment-wise parametric integration.

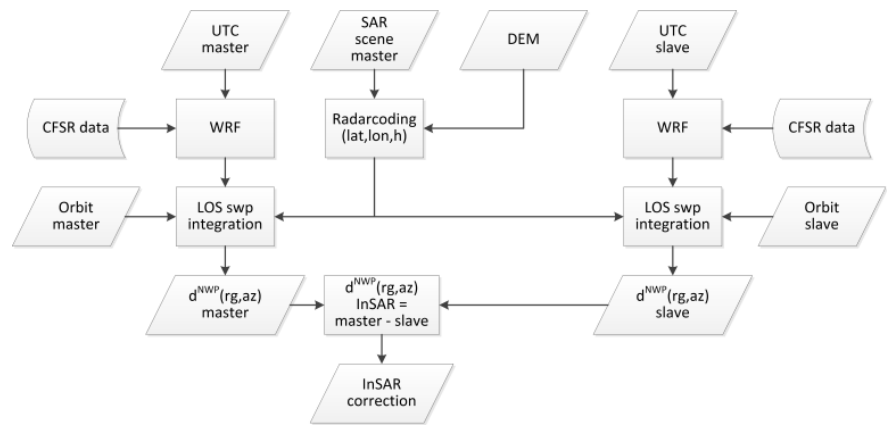

Fig. 8. Process flow diagram of the high resolution APS mitigation (swp: segment-wise parametric).

\section{High Resolution and High Precision APS Mitigation}

NWP hindcasts are solutions of partial differential equations and as a result, they are sensitive to initial atmosphere conditions. Similar to chaotic systems, the uncertainty in a hindcast increases exponentially with elapsed time. A typical example is the wind which is imprecise in speed and direction. As a result, humidity and cold or warm air are misplaced at the simulated SAR acquisition time. In order to deal with this effect, ensembles of $N$ hindcasts $\boldsymbol{\varphi}_{i}^{N W P}$ are computed [17]. $\boldsymbol{\varphi}_{i}^{N W P}$ are APS candidates generated from NWP at different times close to the SAR acquisition time, with different physics parameters, or both. Essentially, a weighted ensemble mean provides the high resolution and high precision APS correction $\widehat{\boldsymbol{\varphi}}^{A P S}$

$$
\widehat{\boldsymbol{\varphi}}^{A P S}=\sum_{i=1}^{N} a_{i} \boldsymbol{\varphi}_{i}^{N W P}[\mathrm{rad}]
$$

The actual implementation estimates additionally to the weights $a_{i}$ a systematic phase component parameterized by the offset $c_{0}$ and the linear trends $c_{1}$ respectively $c_{2}$. Effects not part of the NWP and undefined in InSAR can be modelled by this principle e.g. the phase offset, orbit errors and ionospheric effects. A practical solution is obtained by the constrained minimization

$$
\begin{aligned}
& \underset{\left(a_{i} c_{0,1,2}\right)}{\arg \min }\left\|\boldsymbol{\varphi}^{A P S}-c_{0}-c_{1} \boldsymbol{x}^{P S}-c_{2} \boldsymbol{y}^{P S}-\sum_{i=1}^{N} a_{i} \boldsymbol{\varphi}_{i}^{N W P}\right\|_{1 \mid 2} \\
& \text { subject to: } a_{i} \geq 0 \cap \sum_{i=1}^{N} a_{i}=1
\end{aligned}
$$

$\boldsymbol{\varphi}^{A P S}$ is the APS estimated from PSI at the locations $\left(\boldsymbol{x}^{P S} \boldsymbol{y}^{P S}\right)$. Assuming the deformation phase is much smaller in magnitude and extension compared to the APS, or is known beforehand and is masked, the unwrapped DInSAR phase $\boldsymbol{\varphi}^{\text {DInSAR }}$ can be used likewise. In this case, ensembles of $N_{m}$ and $N_{s}$ APS candidates $\boldsymbol{\varphi}_{i}^{N W P_{m}}$ and $\boldsymbol{\varphi}_{i}^{N W P_{s}}$ are generated for the master and the slave respectively. It is apparent that the interferometric APS $\widehat{\varphi}^{A P S}$ is estimated from the master and the slave ensembles

$$
\widehat{\boldsymbol{\varphi}}^{A P S}=\sum_{i=1}^{N_{m}} a_{i} \boldsymbol{\varphi}_{i}^{N W P_{m}}-\sum_{i=1}^{N_{S}} b_{i} \boldsymbol{\varphi}_{i}^{N W P_{S}}
$$

and the constrained minimization

$$
\begin{aligned}
& \underset{\left(a_{i}, b_{i}, c_{0,1,2}\right)}{\arg \min }\left\|\boldsymbol{\varphi}^{\text {DInSAR }}-c_{0}-c_{1} \boldsymbol{x}^{\text {sel }}-c_{2} \boldsymbol{y}^{\text {sel }}-\widehat{\boldsymbol{\varphi}}^{A P S}\right\|_{1 \mid 2} \\
& \text { subject to: } a_{i} \geq 0 \cap \sum_{i=1}^{N_{m}} a_{i}=1 \cap b_{i} \geq 0 \cap \sum_{i=1}^{N_{S}} b_{i}=1
\end{aligned}
$$

$\boldsymbol{x}^{\text {sel }}$ and $\boldsymbol{y}^{\text {sel }}$ are positions of coherent and deformation masked differential interferogram pixels $\boldsymbol{\varphi}^{\text {DInSAR }}$. The overall process flow diagram of this technique is visualized in Fig. 9.

The choice of $L_{1}$ or $L_{2}$ norm indicated by $\|.\|_{1 \mid 2}$ in Eq. (12) and Eq. (14) depends on the actual test case. For DInSAR input data $\varphi^{\text {DInSAR }}$, the $L_{1}$ norm is recommended because it copes with outliers e.g. small unknown deformation areas and 
IEEE Journal of Selected Topics in Applied Earth Observations and Remote Sensing

small phase unwrapping errors. In case of an APS input estimated from PSI $\boldsymbol{\varphi}^{A P S}$, deformations are compensated already. Now, the $L_{1}$ norm provides a sparse estimate of $a_{i}$ selecting the best fitting APS candidates by close to zeroweights of the other coefficients. Such a solution is preferred if the APS candidates are generated from different physics parameters in WRF. On the other hand, the $L_{2}$ norm is optimal in case of Gaussian errors and is straight forward to implement.

The ensemble size $N, N_{m}$, and $N_{s}$ and the time interval between the ensemble members depend on the WRF horizontal grid size $\Delta x_{W R F}[\mathrm{~km}]$, the maximum wind speed inside a scene $v_{\max }[\mathrm{km} / \mathrm{h}]$, the expected wind speed uncertainty $v_{e r r}[\mathrm{~m} / \mathrm{s}]$ and the WRF hindcast evolution time span $\Delta t_{\text {hets }}[h]$. In practice, the typical uncertainty of wind speed $v_{e r r}$ is about $1 \mathrm{~m} / \mathrm{s}$. For CFSR input data, the temporal sampling is six hours. This explains the maximum hindcast evolution time span $\Delta t_{\text {hets }}^{\max }=6$ hours because the SAR acquisition time is always within this time frame. The hindcast evolution time span is parameterized by the factor $f_{t}$ with $\left\{f_{t} \in \mathbb{R} \mid 0<f_{t} \leq 1\right\}$. It is the hindcast evolution time span parameter which for instance is $1 / 2$ in the middle of the time frame i.e. after three hours and 1 for a radar acquisition after six hours.

$$
\Delta t_{\text {hets }}=f_{t} \cdot \Delta t_{\text {hets }}^{\max }[\mathrm{h}]
$$

Additionally, for tuning the precision, a partial change factor $f_{P C}$ with $\left\{f_{P C} \in \mathbb{R} \mid 0<f_{P C} \leq 1\right\}$ is introduced. It is the fractional amount of the WRF grid area of which the change wants to be observed. In case, the completely changed grid cell becomes an ensemble member, $f_{P C}$ is one. More precise results are obtained with smaller values. E.g. with $f_{P C}=0.5$, ensemble members are added after half of the grid cell is changed. The maximum offset $x_{\text {err }}[\mathrm{km}]$ of humidity and cold or warm air caused by using $v_{\text {hindcast }}$ instead of the true wind speed $v_{\text {true }}$ follows from the fact $v_{\text {hindcast }}=v_{\text {true }} \pm v_{\text {err }}$.

$$
x_{\text {err }}= \pm \frac{3600[\mathrm{~s} / \mathrm{h}]}{1000[\mathrm{~m} / \mathrm{km}]} \cdot v_{\text {err }} \cdot \Delta t_{\text {hets }}[\mathrm{km}]
$$

The maximum wind speed $v_{\max }[\mathrm{km} / \mathrm{h}]$ in the scene translates the offset $x_{\text {err }}[\mathrm{km}]$ into a maximum timing error

$$
t_{\text {err }}= \pm \frac{x_{\text {err }}}{v_{\max }}[\mathrm{h}]
$$

It specifies the required time span around the radar acquisition time $t_{S A R}$ of the WRF hindcast to $\left[t_{S A R}-t_{e r r}, t_{S A R}+t_{e r r}\right]$. The time interval between the ensemble members $\Delta t_{\text {ens }}$ [min] depends on the hindcast grid size $\Delta x_{W R F}[\mathrm{~km}]$, the maximum wind speed $v_{\max }[\mathrm{km} / \mathrm{h}]$ and the partial change factor $f_{P C}$.

$$
\Delta t_{\text {ens }}=60[\mathrm{~min} / \mathrm{h}] \cdot \frac{\Delta x_{W R F}}{v_{\max }} \cdot f_{P C}[\mathrm{~min}]
$$

The ensemble size $N$ (also $N_{m}$, and $N_{s}$ ) follows from the maximum timing error $t_{e r r}[\mathrm{~h}]$ and the time interval between the ensemble members $\Delta t_{\text {ens }}[\mathrm{min}]$ with $\lceil$.$\rceil being the ceiling$ function.

$$
\begin{aligned}
N & =\left\lceil 60[\mathrm{~min} / \mathrm{h}] \cdot \frac{2 \cdot t_{\text {err }}}{t_{\text {ens }}}\right\rceil \\
& =\left\lceil\frac{36[\mathrm{~s} / \mathrm{h}]}{5[\mathrm{~m} / \mathrm{km}]} \cdot \frac{f_{t}}{f_{P C}} \cdot \frac{\Delta t_{\text {hets }}^{\text {max }} \cdot v_{\text {err }}}{\Delta x_{W R F}}\right\rceil
\end{aligned}
$$

For the examples in Table $\mathrm{I}, f_{P C}=1$ and $f_{t}=1$ are chosen.

TABLE I

Ensemble size $N$ and the time interval between the ensemble members.

\begin{tabular}{|c|c|c|c|c|}
\hline & \multicolumn{2}{|c|}{$\Delta x_{\text {WRF }}=3 \mathrm{~km}$} & \multicolumn{2}{c|}{$\Delta x_{\text {WRF }}=1 \mathrm{~km}$} \\
\hline & $\Delta \mathrm{t}_{\text {ens }}[\mathrm{min}]$ & $\mathrm{N}$ & $\Delta \mathrm{t}_{\text {ens }}[\mathrm{min}]$ & $\mathrm{N}$ \\
\hline $\mathrm{v}_{\max }=10 \mathrm{~km} / \mathrm{h}$ & 18.0 & 15 & 6.0 & 44 \\
\hline $\mathrm{v}_{\max }=20 \mathrm{~km} / \mathrm{h}$ & 9.0 & 15 & 3.0 & 44 \\
\hline $\mathrm{v}_{\max }=30 \mathrm{~km} / \mathrm{h}$ & 6.0 & 15 & 2.0 & 44 \\
\hline $\mathrm{v}_{\max }=40 \mathrm{~km} / \mathrm{h}$ & 4.5 & 15 & 1.5 & 44 \\
\hline $\mathrm{v}_{\max }=50 \mathrm{~km} / \mathrm{h}$ & 3.6 & 15 & 1.2 & 44 \\
\hline $\mathrm{v}_{\max }=60 \mathrm{~km} / \mathrm{h}$ & 3.0 & 15 & 1.0 & 44 \\
\hline
\end{tabular}

A suggestion of Richard Bamler is relaxing the constraints in Eq. (12) and Eq. (14) to $\sum_{i=1}^{N_{m}} a_{i} \cong 1$ and $\sum_{i=1}^{N_{s}} b_{i} \cong 1$. As a consequence, the APS estimation can cope with biased hindcasts. The actual implementation follows this suggestion and the examples presented in the following section include this feature.

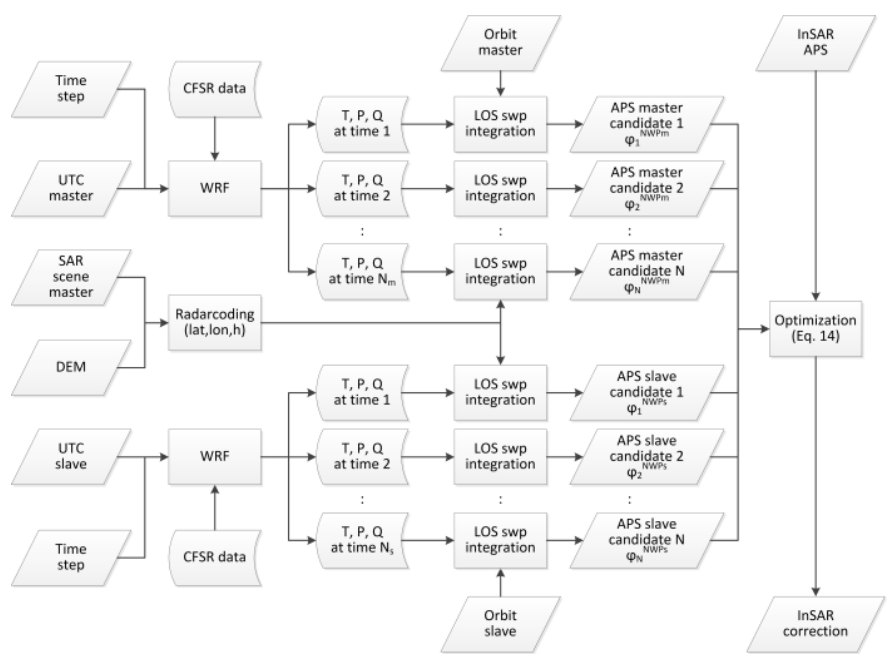

Fig. 9. Process flow diagram of the high resolution and high precision APS mitigation (swp: segment-wise parametric).

\section{RESULTS}

\section{A. Master selection support}

Fig. 10 compares three geocoded zenith wet delay products taken from ERS acquisitions (black rectangle). The mean values in the scenes areas are $105.7 \mathrm{~mm}$ (left) $96.5 \mathrm{~mm}$ (middle) and $37.1 \mathrm{~mm}$ (right). In this example, the right acquisition taken on Mar 23, 1998 is a better master scene. It follows from two facts. First, more humidity impacts the radar 
IEEE Journal of Selected Topics in Applied Earth Observations and Remote Sensing

wave propagation in the two left acquisitions (from Sep 25, 1995 and Jan 8, 1996) as the mean is a substitute for the total amount of humidity. And second, humidity predicted from WRF follows a power law which explains the expected higher variation on the high resolution InSAR scale. It is based on wind transport plus mass and energy conservation equations. In reality, water vapor is mainly contained in the near-ground surface troposphere where a strong turbulent mixing process occurs [32]. As a consequence, it is spatially heterogeneous. However, WRF cannot model turbulent processes. Fig 11 confirms the power law characteristic of the WRF output and the previous master scene selection by the visualization of the respective semivariograms.
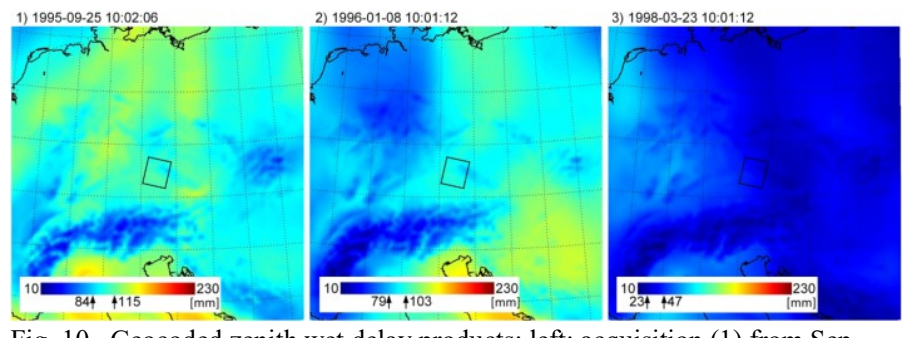

Fig. 10. Geocoded zenith wet delay products; left: acquisition (1) from Sep 25, 1995; middle: acquisition (2) from Jan 8, 1996 and right: acquisition (3) from Mar 23, 1998.

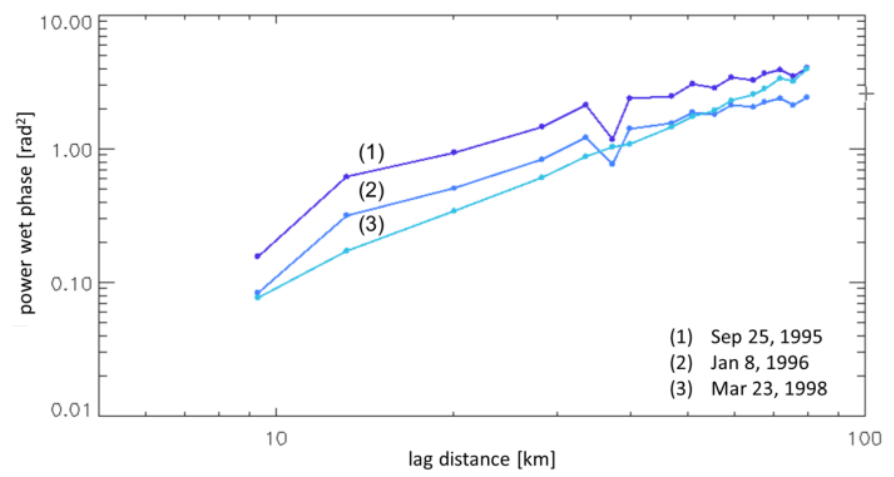

Fig. 11. Semivariograms of the geocoded zenith wet delay for the examples from Fig. 10.

\section{B. Vertical stratification mitigation}

Fig. 4 (right) visualizes a vertical stratification mitigation phase. It corrects each interferogram sample with an individual value. In plain areas, only little correction is applied. The correction is very smooth in such areas because it uses the NWP hindcast on a very coarse grid only and as a consequence, local atmosphere effects are not mitigated. However, this mitigation is very valuable if the relative estimation spans a height difference. Applying the stratification mitigation, more PSs in mountainous areas can be connected by relative estimates. Fig. 12 provides an example. The periodogram $|\gamma|$ [unitless]

$$
\begin{aligned}
& \left|\gamma\left(\Delta v_{P S 1,2}, \Delta h_{P S 1,2}\right)\right| \\
& =\mid \frac{1}{K} \sum_{k=1}^{K} e^{j \cdot\left(\varphi_{k}^{\text {DInSARPS1 }}-\varphi_{k}^{\text {DInSAR }_{P S 2}}\right) \cdot e^{-j \cdot m o d e l} \mid}
\end{aligned}
$$

represents the likelihood of an estimate [12] and is visualized for uncorrected (upper left)

$$
\operatorname{model}_{u}=B_{k} \cdot \Delta h_{P S 1,2}+T_{k} \cdot \Delta v_{P S 1,2}[\mathrm{rad}]
$$

and stratification corrected (upper right)

$$
\begin{aligned}
\text { model }_{c}= & B_{k} \cdot \Delta h_{P S 1,2}+T_{k} \cdot \Delta v_{P S 1,2}+ \\
& \left(\varphi_{k}^{\left.A P S_{P S 1}-\varphi_{k}^{A P S_{P S 2}}\right)[\mathrm{rad}]}\right.
\end{aligned}
$$

data. In the equations above, the relative (i.e. between PS1 and PS2) DEM update $\Delta h_{P S 1,2}[\mathrm{~m}]$ and the relative deformation rate $\Delta v_{P S 1,2}[\mathrm{~mm} / \mathrm{yr}]$ are the PSI estimates. $K$ is the number of differential interferograms $\varphi_{k}^{D I n S A R}[\mathrm{rad}]$ in the stack. $\varphi_{k}^{\text {DInSAR }_{P S 1}}$ and $\varphi_{k}^{\text {DInSAR }_{P S 2}}$ denote the data from PS1 and PS2 respectively. $B_{k}[\mathrm{rad} / \mathrm{m}]$ is the respective height to phase conversion factor and $T_{k}\left[\mathrm{rad} \mathrm{yr} \mathrm{mm}^{-1}\right]$ is the respective velocity to phase conversion factor with the corresponding velocity expressed in $\mathrm{mm} / \mathrm{yr} . \varphi_{k}^{A P S_{P S 1}}$ and $\varphi_{k}^{A P S_{P S 2}}$ are the interferometric stratification corrections for the interferogram with index $k$ at the location of PS1 and PS2 respectively. Only in the corrected data (upper right), the peak can unambiguously be detected. The coherence improvement depending on the relative height difference has a quadratic characteristic. It is plotted in Fig. 13. For a typical ERS stack, the coherence improves approximately by $10 \%$ for a height difference of $200 \mathrm{~m}$ and by $20 \%$ for $300 \mathrm{~m}$.

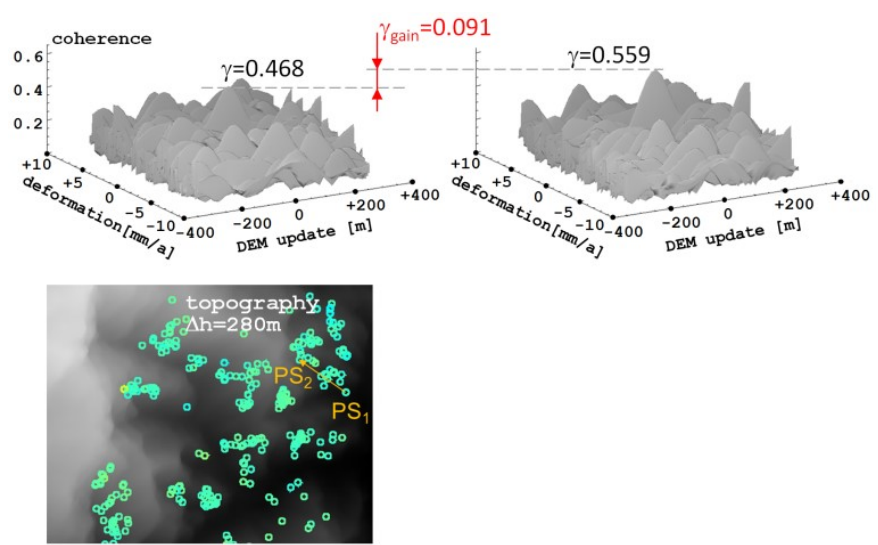

Fig. 12. Top row: periodograms demonstrating an improved estimation, bottom figure: distribution of the respective PSs in a mountainous area (topography is background).

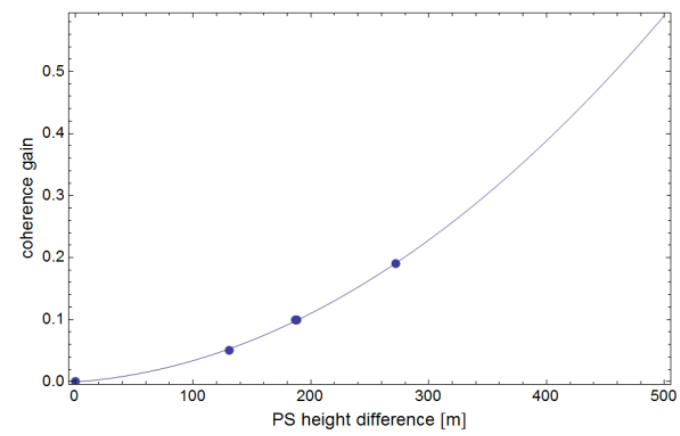

Fig. 13. Coherence improvement depending on height difference. 
IEEE Journal of Selected Topics in Applied Earth Observations and Remote Sensing

\section{High resolution APS mitigation}

Fig. 14 right provides an example for an interferometric APS calculated from NWP hindcasts with $3 \mathrm{~km}$ resolution. The test site is in the North German lowlands with very little topography variation. Now, the mitigation phase shows local tropospheric effects. Fig. 15 provides a semivariogram of the residual phase after a PSI processing with uncompensated (solid line) and compensated (dotted line) data. In this example, the SNR of the interferometric data is doubled. It results directly from the high resolution atmosphere correction because of the lack of topography. The semivariogram demonstrates the improvement for a single interferogram. In contrast, the periodogram includes all interferograms and can indicate the improvement for all scenes. Fig. 16 compares the

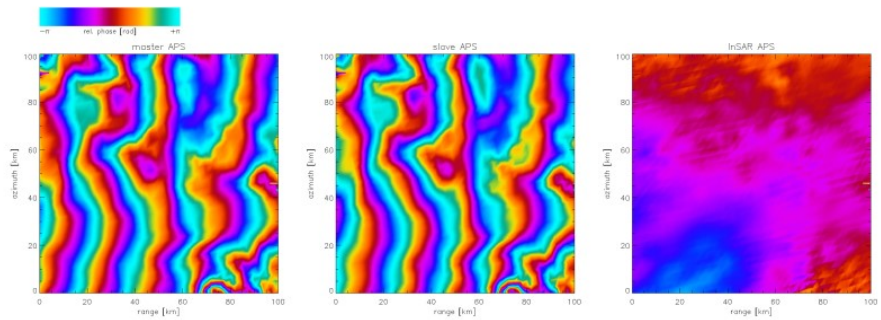

Fig. 14. APS estimated from $3 \mathrm{~km} \times 3 \mathrm{~km} \mathrm{NWP}$ (WRF). Left: master APS, middle: slave APS, right: interferometric APS.

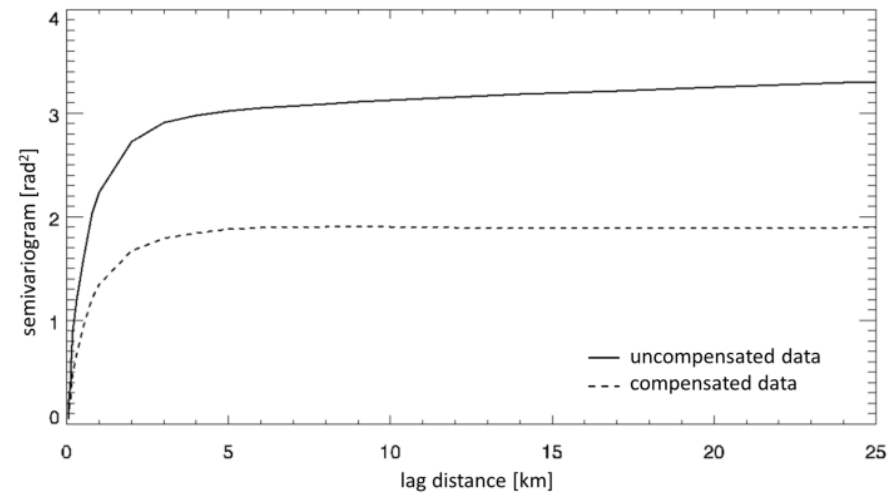

Fig. 15. Semivariogram of uncompensated (solid line) and compensated (dotted line) data.

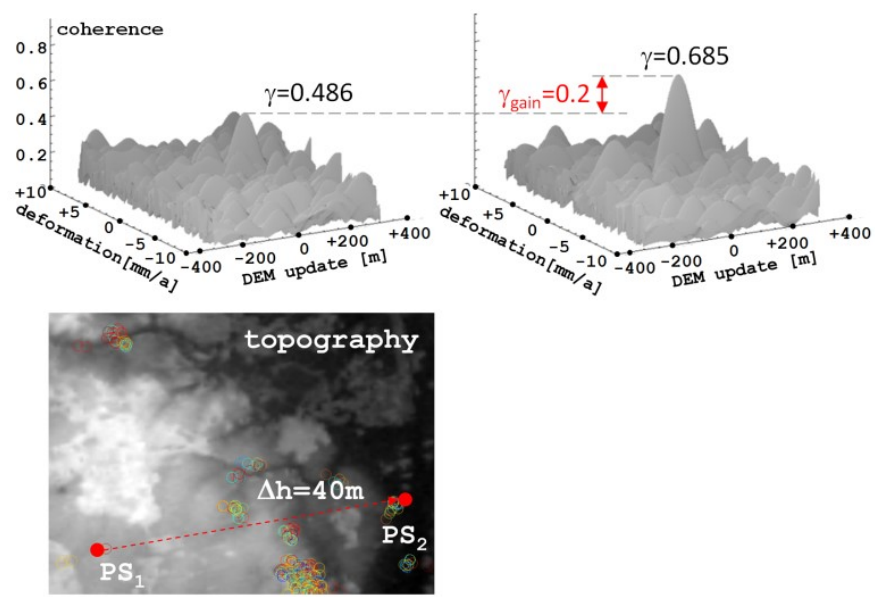

Fig. 16. Top row: periodograms demonstrating an improved estimation, bottom figure: distribution of the respective PSs in the test site (topography is background). periodograms (Eq. (20) - (22)) for the uncompensated (upper left) and compensated (upper right) data. The coherence improves from 0.486 to 0.685 which corresponds to an SNR improvement of about $3.6 \mathrm{~dB}$.

\section{High resolution and high precision APS mitigation}

The weighted ensemble compensates best for the tropospheric effect. The reason is the use of DInSAR or PSI data to correct for the NWP uncertainties. Fig. 17 shows an ERS example for an area of about $100 \mathrm{~km} \times 100 \mathrm{~km}$ with the interferometric data (top row) and the estimated components (middle and bottom rows). In this test case, the residual phase (i.e. the APS) of the input interferogram spans more than a phase cycle (upper left figure). However, the compensated interferogram (upper right figure) is within half a phase cycle. In the upper middle figure, the estimated InSAR APS is corrected but not the residual trend caused e.g. by an orbit error or the ionospheric effect.

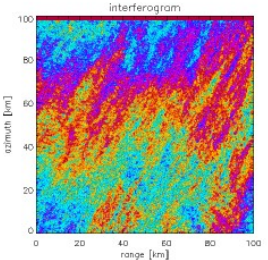

range $[\mathrm{km}]$
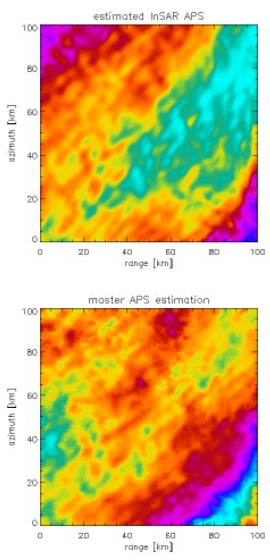

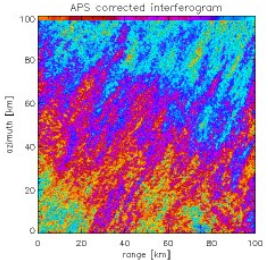

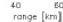
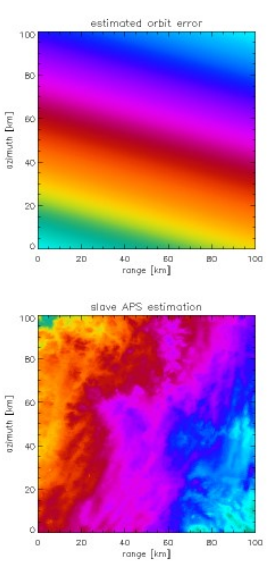

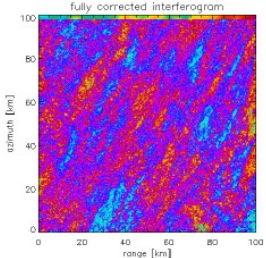

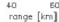
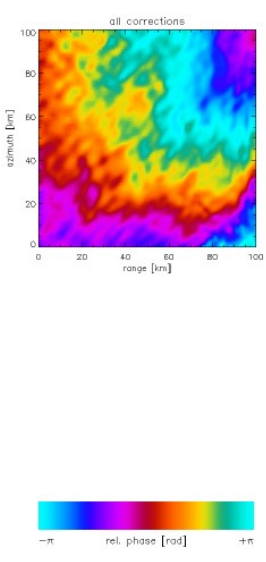

Fig. 17. Estimated components for an area of $100 \mathrm{~km} \times 100 \mathrm{~km}$.

In this example, the ensemble of APS candidates is generated from WRF hindcasts with $1 \mathrm{~km} \times 1 \mathrm{~km}$ resolution and time separations of 10 minutes from each other close to the SAR acquisition time. Fig. 18 visualizes a subset of the used master APS candidates. To illustrate the change of troposphere, a time separation of 20 minutes and the wet component solely, i.e. without the dominant dry effect, is presented. The weighted combination of the APS candidates provides the estimated APS of the master (Fig. 17 bottom row left figure), of the slave (bottom row middle figure) and finally of the differential interferogram (middle row left figure). All these APS estimates show the high resolution spatial pattern of humidity. The semivariogram provides the statistical figure on the SNR improvement and is provided in Fig. 19. A solid line is used for the uncompensated and a dotted line for the APS mitigated data. First, a small variance is observed for both data 
sets. It results from the selected test case. To better demonstrate the difficult compensation of the wet effect, a one day separated ERS-TANDEM acquisition pair and a test site with only little topography (the North German lowlands) are used. The scenes are acquired in summer on Jul 6, 1996 and Jul 7, 1996. This approach results in small dry and vertical stratification components confirmed in the semivariogram. Second, Fig. 19 shows that the estimation can mitigate the wet troposphere effect down to a resolution of $5 \mathrm{~km}$. By means of larger ensembles of NWP candidates and with better spatial resolution, this characteristic can be improved.
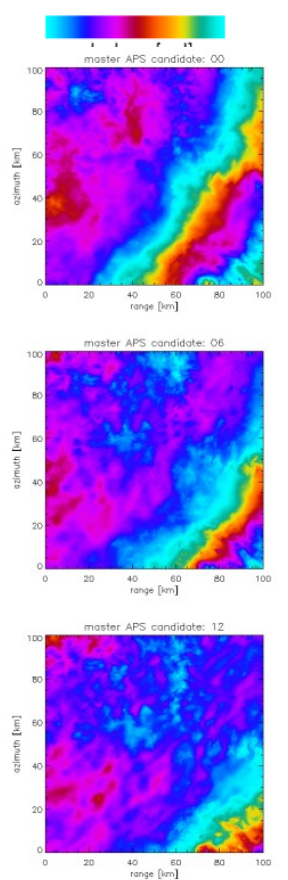

Fig. 18. Visualization of a subset of the master APS candidates (time separation of 20 minutes and wet component solely, i.e. without dry effect).

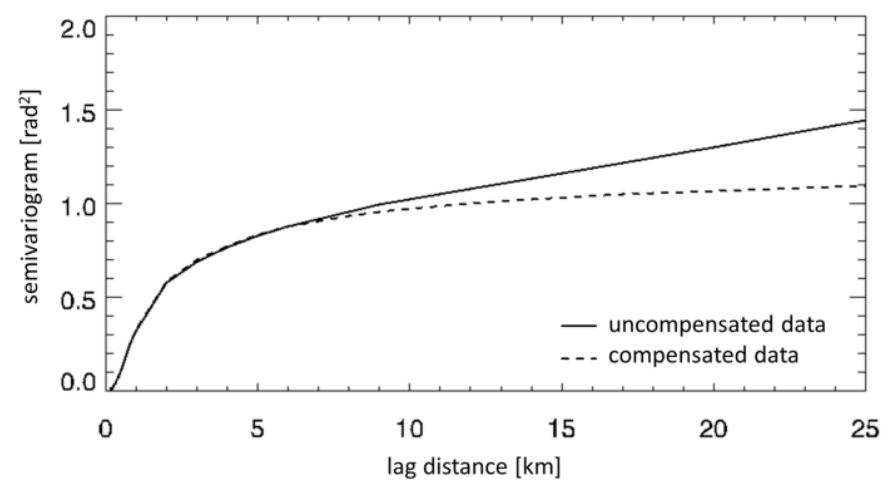

Fig. 19. Semivariogram of uncompensated (solid line) and compensated (dotted line) data.

\section{DISCUSSION}

Four options to implement atmosphere mitigation are presented, namely the master selection support, the vertical stratification mitigation, the high resolution APS mitigation and the high resolution and high precision APS mitigation. All have different characteristics e.g. complexity of implementation, requirements on the hardware and input data, mitigation precision and spatial resolution.
The choice of the quadrature method for Eq. (2) affects the precision and computational effort. Both are essential aspects of operational systems and an acceptable trade-off specific for each mitigation method needs to be implemented. The Newton-Cotes integration, used in the master selection support, integrates a tabulated set of data. Simplified, the WRF output parameters are first transformed into the scaledup refractivity on the given $3 \mathrm{D}$-grid and then integrated. Additionally, the Newton-Cotes algorithm fits polynomials for the integration assuming the refractivity can be modelled by a polynomial in vertical direction. For the master selection support, these simplifications can be tolerated because the computed wet effect is not applied directly on the InSAR data. In contrast to Newton-Cotes, the Gauss-Kronrod quadrature, implemented in the vertical stratification mitigation, is a nested iterative method which varies the step size and the polynomial order based on an error estimate. As a consequence, it requires 3D-interpolation in the given 3D-grid of WRF. Due to the fact the Smith Weintraub Eq. (1) is nonlinear, the WRF output parameters $P_{t o t}, T_{K}$ and $Q_{k g / k g}$ need to be interpolated and transformed into the scaled-up refractivity in each integration step. It is more precise, however much more computationally intensive compared to Newton Cotes. For the high resolution mitigation methods it is inexpedient. The newly developed segment-wise parametric integration solves the performance problem.

In practice, the master selection support and basic vertical stratification mitigation can be implemented using coarse resolution atmosphere data directly (e.g. CFSR or ECMWF) without the need of a weather model execution. This can reduce the CPU and memory cost compared to the other techniques. However, for all methods, the mitigation performance depends on the spatial and temporal sampling of the hindcast. Simply, the master selection support is less demanding in the spatial sampling. In this technique, the predicted wet effect is no longer considered after selecting an appropriate master scene based on it. Currently, the implementation in the TEMP framework generates WRF hindcasts with $3 \mathrm{~km}$ horizontal resolution because of only moderate hardware requirements. Nowadays, hindcasts with 1 $\mathrm{km}$ resolution are easily available using fast CPUs and CFSR input data. The high horizontal resolution is an important advantage and the basis for a better mitigation compared to the sparse sampling of the alternative techniques (B, C, D and E). In vertical direction, the number of levels is fixed to 50 in the developed processing system and the demonstrated examples. Abrupt changes in temperature and water vapor mixing ratio are physically realistic in this direction. In practice, the vertical sampling in the WRF model should allow the representation and physical modelling of these abrupt effects. Tests have demonstrated, the hindcast does not improve with more than 50 levels [24] and as a consequence increasing the number of levels does not affect the mitigation performance.

The master selection support, the vertical stratification mitigation and the high resolution APS mitigation are independent of interferometric data whereas, the high resolution and high precision APS mitigation utilizes the 
interferometric phase in order to correct for the NWP uncertainties. As a consequence, the high resolution and high precision APS mitigation is available in parallel to a PSI processsing only and is not suitable for an independent service. As shown, it provides the best troposphere effect mitigation on a price of being computationally more costly. The high resolution APS mitigation and the high resolution and high precision APS mitigation are considered advanced methods due to the mitigation of local troposphere effects with high resolution. At least one of the two should be implemented in an operational system.

\section{CONCLUSION}

Troposphere effect mitigation is a key methodology in order to improve the InSAR measurement precision, support PSI wide area processing and InSAR applications in mountainous areas. For this reason, the techniques are relevant for completed (e.g. ERS-1/2, RADARSAT-1), current (e.g. Sentinel-1, TerraSAR-X) and proposed (e.g. Tandem-L) SAR missions. In practice, all four presented methods improve the SNR of the interferometric data. Compared to other techniques, the NWP based methods overcome limitations of spatial sampling, global data availability and temporal interpolation. For operational InSAR systems, it is recommended to implement the master selection support and at least one of the advanced methods i.e. high resolution APS mitigation or high resolution and high precision APS mitigation.

\section{ACKNOWLEDGEMENT}

I appreciate the work of the anonymous reviewers and I am grateful that they have read the manuscript critically and suggested significant improvements.

\section{REFERENCES}

[1] D. Massonnet, K. Feigl, M. Rossi and F. Adragna, "Radar interferometric mapping of deformation in the year after the Landers earthquake," Nature, vol. 369, pp. 227-230, 1994.

[2] R. Goldstein, "Atmospheric limitations to repeat-track radar interferometry," Geophysical Research Letters, vol. 22, pp. 2517-2520, 1995.

[3] H. Tarayre and D. Massonnet, "Atmospheric Propagation heterogeneities revealed by ERS-1 interferometry," Geophysical Research Letters, vol. 23, no. 9, pp. 989-992, 1996.

[4] H. A. Zebker, P. A. Rosen and S. Hensley, "Atmospheric effects in interferometric synthetic aperture radar surface deformation and topographic maps," Journal of Geophysical Research: Solid Earth, vol. 102, no. B4, pp. 7547-7563, 04 April 1997.

[5] R. F. Hanssen, "Atmospheric heterogeneities in ERS tandem SAR interferometry," Delft University, Delft, the Netherlands, DEOS Report No.98.1, 1998.

[6] S. Williams, Y. Bock and P. Fang, "Integrated satellite interferometry: Tropospheric noise, GPS estimates and implications for interferometric synthetic aperture radar products," Journal of Geophysical Research: Solid Earth, vol. 103, no. B11, pp. 27051-27067, 1998.

[7] R. F. Hanssen, Radar Interferometry: Data Interpretation and Error Analysis, Dordrecht: Kluwer Academic Publishers, 2001.

[8] F. Meyer, R. Bamler, N. Jakowski and T. Fritz, "The Potential of LowFrequency SAR Systems for Mapping Ionospheric TEC Distributions," IEEE Geoscience and Remote Sensing Letters, pp. 560-564, 23 October 2006.

[9] P. Rosen, S. Hensley and C. Chen, "Measurement and mitigation of the ionosphere in L-band Interferometric SAR data," in 2010 IEEE Radar
Conference, 2010.

[10] F. Brunner and M. Gu, "An improved model for the dual frequency ionospheric correction of GPS observations," Manuscripta Geodaetica, vol. 16, pp. 205-214, January 1991.

[11] A. Ferretti, C. Prati and F. Rocca, "Permanent scatterers in SAR interferometry," IEEE 1999 International Geoscience and Remote Sensing Symposium. IGARSS'99 (Cat. No.99CH36293), vol. 3, pp. 15281530, June 1999.

[12] A. Ferretti, C. Prati, F. Rocca, "Permanent Scatterers in SAR Interferometry," IEEE Transactions on Geoscience and Remote Sensing, vol. 38, pp. $2202-2212.2000$.

[13] A. Ferretti, C. Prati and F. Rocca, "Permanent scatterers in SAR interferometry," IEEE Transactions on Geoscience and Remote Sensing, vol. 39, no. 1, pp. 8-20, January 2001.

[14] P. Berardino, G. Fornaro, R. Lanari and E. Sansosti, "A new algorithm for surface deformation monitoring based on small baseline differential SAR interferograms," IEEE Transactions on Geoscience and Remote Sensing, vol. 40, no. 11, pp. 2375-2383, November 2002.

[15] T. R. Emardson, M. Simons and F. H. Webb, "Neutral atmospheric delay in interferometric synthetic aperture radar applications: Statistical description and mitigation," Journal of Geophysical Research: Solid Earth, vol. 108, no. B5, pp. 2231-2238, 2003.

[16] F. Rocca, "Diameters of the orbital tubes in long-term interferometric SAR surveys," IEEE Geoscience and Remote Sensing Letters, vol. 1, no. 3, pp. 224-227, July 2004.

[17] F. Ulmer and N. Adam, "A Synergy Method to Improve Ensemble Weather Predictions and Differential SAR Interferograms," ISPRS Journal of Photogrammetry and Remote Sensing, vol. 109, pp. 98-107, 2015.

[18] A. Bonforte, A. Ferretti, C. Prati, G. Puglisi and F. Rocca, "Calibration of atmospheric effects on SAR interferograms by GPS and local atmosphere models: first results," Journal of Atmospheric and SolarTerrestrial Physics, vol. 63, pp. 1343-1357, 2001.

[19] F. Onn and H. Zebker, "Correction for interferometric synthetic aperture radar atmospheric phase artifacts using time series of zenith wet delay observations from a GPS network," Journal of Geophysical Research: Solid Earth, vol. 111, no. B9, September 2006.

[20] F. Beauducel, P. Briole and J.-L. Froger, "Volcano-wide fringes in ERS synthetic aperture radar interferograms of Etna (1992-1998): Deformation or tropospheric effect?," Journal of Geophysical Research: Solid Earth, vol. 105, no. B7, pp. 16391-16402, 2000.

[21] J. Wicks, W. Charles, D. Dzurisin, S. Ingebritsen, W. Thatcher, Z. Lu and J. Iverson, "Magmatic activity beneath the quiescent Three Sisters volcanic center, central Oregon Cascade Range, USA," Geophysical Research Letters, vol. 29, no. 7, pp. 26-1-26-4, 2002.

[22] J. Elliott, J. Biggs, B. Parsons and T. Wright, "InSAR slip rate determination on the Altyn Tagh Fault, northern Tibet, in the presence of topographically correlated atmospheric delays," Geophysical Research Letters, vol. 35, no. 12, pp. 1-5, 2008.

[23] Y. N. Lin, M. Simons, E. A. Hetland, P. Muse and C. DiCaprio, "A multiscale approach to estimating topographically correlated propagation delays in radar interferograms," Geochemistry, Geophysics, Geosystems, vol. 11, no. 9, pp. 1-17, 2 September 2010.

[24] N. Adam, „Algorithmic PSI Improvement in Mountainous Areas by Atmosphere Mitigation", DLR Oberpfaffenhofen, Oberpfaffenhofen, Germany, Tech. Rep TFX-TN. Nov. 2014. [Online]. Available: http://elib.dlr.de/ 95190/1/AtmosphereMitigation v3.10.pdf

[25] D. Bekaert, R. Walters, T. Wright, A. Hooper and D. Parker, "Statistical comparison of InSAR tropospheric correction techniques," Remote Sensing of Environment, vol. 170, pp. 40-47, 1 December 2015.

[26] H. Liang, L. Zhangl, X. Ding, Z. Lu and X. Lil, "A Joint Model for Isolating Stratified Tropospheric Delays in Multi-Temporal Insar," IGARSS 2018 - 2018 IEEE International Geoscience and Remote Sensing Symposium, pp. 2258-2261, July 2018.

[27] H. Liang, L. Zhang, X. Ding, Z. Lu and X. Li, "Toward Mitigating Stratified Tropospheric Delays in Multitemporal InSAR: A Quadtree Aided Joint Model," IEEE Transactions on Geoscience and Remote Sensing, vol. 57, no. 1, pp. 291-303, January 2019.

[28] M. Bevis, S. Businger, T. A. Herring, C. Rocken, R. A. Anthes and R. H. Ware, "GPS meteorology: Remote sensing of atmospheric water vapor using the global positioning system," Journal of Geophysical Research: Atmospheres, vol. 97, no. D14, pp. 15787-15801, 20 October 1992.

[29] V. Bruno, M. Aloisi, A. Bonforte, G. Immè and G. Puglisi, "Atmospheric anomalies over Mt. Etna using GPS signal delays and tomography of radio wave velocities," Annals of Geophysics, vol. 50, no. 
IEEE Journal of Selected Topics in Applied Earth Observations and Remote Sensing

2, pp. 267-282, 2 April 2007.

[30] C. Yu, N. T. Penna and Z. Li, "Generation of real-time mode highresolution water vapor fields from GPS observations," J. Geophys. Res. Atmos, vol. 122, p. 2008-2025, 26 January 2017.

[31] C. Yu, Z. Li and N. T. Penna, "Interferometric synthetic aperture radar atmospheric correction using a GPS-based iterative tropospheric decomposition model," Remote Sensing of Environment, vol. 204, pp. 109-121, 2018.

[32] X.-1. Ding, Z.-w. Li, J.-j. Zhu, G.-c. Feng and J.-p. Long, "Atmospheric Effects on InSAR Measurements and Their Mitigation," sensors, pp. 5426-5448, 3 September 2008.

[33] M.-P. Doin, C. Lasserre, G. Peltzer, O. Cavalié and C. Doubre, "Corrections of stratified tropospheric delays in SAR interferometry: Validation with global atmospheric models," Journal of Applied Geophysics, p. 35-50, 25 March 2009.

[34] R. Jolivet, R. Grandin, C. Lasserre, M.-P. Doin and G. Peltzer, "Systematic InSAR tropospheric phase delay corrections from global meteorological reanalysis data," Geophysical Research Letters, p. L17311, 14 September 2011.

[35] R. Jolivet, A. P. Shanker, N. Y. Lin, M. Simons, M.-P. Doin, G. Peltzer and $\mathrm{Z}$. Li, "Improving InSAR geodesy using Global Atmospheric Models," Journal of Geophysical Research: Solid Earth, pp. 2324-2341, 2014.

[36] A. L. Parker, J. Biggs , R. J. Walters , S. K. Ebmeier, T. J. Wright, N. A. Teanby and Z. Lu, "Systematic assessment of atmospheric uncertainties for InSAR data at volcanic arcs using large-scale atmospheric models: Application to the Cascade volcanoes, United States," Remote Sensing of Environment, vol. 170, pp. 102-114, 1 December 2015.

[37] H. Fattahi and F. Amelung, "InSAR bias and uncertainty due to the systematic and stochastic tropospheric delay," Journal of Geophysical Research: Solid Earth, vol. 120, no. 12, pp. 8758-8773, 27 November 2015.

[38] Z. Li, W. Xu, G. Feng, J. Hu, C. Wang, X. Ding and J. Zhu, "Correcting atmospheric effects on InSAR with MERIS water vapour data and elevation-dependent interpolation model," Geophysical Journal International, vol. 189, p. 898-910, 1 May 2012.

[39] G. Wadge, P. W. Webley, I. N. James, R. Bingley, A. Dodson, S. Waugh, T. Veneboer, G. Puglisi, M. Mattia, D. Baker, S. C. Edwards, S. J. Edwards and P. J. Clarke, "Atmospheric models, GPS and InSAR measurements of the tropospheric water vapour field over Mount Etna," Geophysical Research Letters, vol. 29, no. 19, pp. 11-1 - 11-4, 3 October 2002.

[40] P. Webley, G. Wadge and I. N. James, "Determining radio wave delay by non-hydrostatic atmospheric modelling of water vapour over mountains," Physics and Chemistry of the Earth, vol. 29, no. 2-3, p. 139-148, 2004.

[41] J. Foster, B. Brooks, T. Cherubini, C. Shacat, S. Businger and C. L. Werner, "Mitigating atmospheric noise for InSAR using a high resolution weather model," Geophysical Research Letters, vol. 33, no. 16, p. L16304, 17 August 2006.

[42] R. Holley, G. Wadge, M. Zhu, (2007, April). "New insights into the nature and effects of the water vapour field on InSAR measurements over Etna." Presented at FRINGE 2007. [Online]. Available: http://earth.esa.int/fringe07/participants/159/pres_159 holley.pdf

[43] C. Yu, Z. Li, N. T. Penna and P. Crippa, "Generic Atmospheric Correction Model for Interferometric Synthetic Aperture Radar Observations," Journal of Geophysical Research: Solid Earth, vol. 123, p. 9202-9222, 12 September 2018.

[44] J. Ruiz Rodon, A. Broquetas, A. Monti Guarnieri and F. Rocca, "Geosynchronous SAR Focusing With Atmospheric Phase Screen Retrieval and Compensation," IEEE Trans. Geoscience and Remote Sensing, vol. 51, no. 8, pp. 4397-4404, August 2013.

[45] D. Li, M. Rodriguez-Cassola, P. Prats-Iraola, Z. Dong, M. Wu and A. Moreira, "Modelling of tropospheric delays in geosynchronous synthetic aperture radar," Science China Information Sciences, vol. 60, online, June 2017.

[46] X. Dong, J. Hu, C. Hu, T. Long, Y. Li and Y. Tian, "Modeling and Quantitative Analysis of Tropospheric Impact on Inclined Geosynchronous SAR Imaging," Remote Sensing, vol. 11, no. 7, 3 April 2019.

[47] D. Perissin, F. Rocca, N. Pierdicca, E. Pichelli, D. Cimini, G. Venuti and B. Rommen, "Mitigation of Atmospheric Delay in InSAR: the ESA METAWAVE Project," 2011 IEEE International Geoscience and Remote Sensing Symposium, pp. 2558-2561, 24-29 July 2011.

[48] D. Cimini, N. Pierdicca, E. Pichelli, R. Ferretti, V. Mattioli, S. Bonafoni, M. Montopoli and D. Perissin, "On the accuracy of integrated water vapor observations and the potential for mitigating electromagnetic path delay error in InSAR," Atmospheric Measurement Techniques, vol. 5, no. 5, pp. 1015-1030, 1052012.

[49] M. Eineder, U. Balss, X. Cong, S. Suchandt, C. Gisinger, H. Runge, "Adding Precise Wave-Propagation Information and Geodetic Corrections to Standard SAR Products," in Proc. CEOS SAR Calibration and Validation Workshop, Noordwijk, the Netherlands, 27.-29. Oct. 2015.

[50] P. von Allmen, E. Fielding, E. Fishbein, Z. Li, Z. Xing, L. Pan. (2011, September) "OSCAR: Online Services for Correcting Atmosphere in Radar." Presented at FRINGE 2011. [Online]. Available: https://earth.esa.int/documents/10174/1566700/Allmen_FRINGE2011.p df

[51] A.C. Kalia, M. Frei, T. Lege, "A Copernicus downstream-service for the nationwide monitoring of surface displacements in Germany," Remote Sensing of Environment, vol. 202, pp. 234-249, 2017.

[52] National Center for Atmospheric Research (NCAR), "Weather Research and Forecasting Model." [Online]. Available: https://www.mmm.ucar.edu/weather-research-and-forecasting-model

[53] E. K. Smith and S. Weintraub, "The constants in the equation for atmospheric refractive index at radio frequencies," Proc. IRE, Bd. 41, pp. 1035-1037, 1953.

[54] J. M. Rüeger, (2002, April). "Refractive Index Formulae for Radio Waves",Presented at XXII FIG International Congress. [Online]. Available:

https://www.fig.net/resources/proceedings/fig_proceedings/fig_2002/Js2 8/JS28 rueger.pdf

[55] S. Saha, S. Moorthi, H. Pan, X. Wu, J. Wang, S. Nadiga, P. Tripp, R. Kistler, J. Woollen, D. Behringer, H. Liu, D. Stokes, R. Grumbine, G. Gayno, J. Wang, Y. Hou, H. Chuang, H. H. Juang, J. Sela, M. Iredell, R. Treadon, D. Kleist, P. Van Delst, D. Keyser, J. Derber, M. Ek, J. Meng, H. Wei, R. Yang, S. Lord, H. van den Dool, A. Kumar, W. Wang, C. Long, M. Chelliah, Y. Xue, B. Huang, J. Schemm, W. Ebisuzaki, R. Lin, P. Xie, M. Chen, S. Zhou, W. Higgins, C. Zou, Q. Liu, Y. Chen, Y. Han, L. Cucurull, R. W. Reynolds, G. Rutledge, and M. Goldberg. "NCEP Climate Forecast System Reanalysis (CFSR) 6-hourly Products, January 1979 to December 2010." Research Data Archive at the National Center for Atmospheric Research, Computational and Information Systems Laboratory, Boulder, CO, 2010, [Online]. Available: https://doi.org/10.5065/D69K487J

[56] S. Saha, S. Moorthi, X. Wu, J. Wang, S. Nadiga, P. Tripp, D. Behringer, Y. Hou, H. Chuang, M. Iredell, M. Ek, J. Meng, R. Yang, M. P. Mendez, H. van den Dool, Q. Zhang, W. Wang, M. Chen, and E. Becker. "NCEP Climate Forecast System Version 2 (CFSv2) 6-hourly Products." Research Data Archive at the National Center for Atmospheric Research, Computational and Information Systems Laboratory, Boulder, CO, 2011, [Online]. Available: https://doi.org/10.5065/D61C1TXF

[57] B. M. Kampes, Radar Interferometry Persistent Scatterer Technique, DOI: $10.1007 / 978-1-4020-4723-7$ ed., vol. 12, The Netherlands: Springer Netherlands, 2006, pp. XXI, 211.

Nico Adam received the Diploma degree in electrical engineering and telecommunication science from the University of Rostock, Germany in 1995.

He has been with the German Aerospace Center (DLR), Oberpfaffenhofen, since 1995, where he manages SAR interferometry projects at DLR's Remote Sensing Technology Institute. His research interests include signal processing in the persistent scatterer interferometry framework, the use of numerical weather models to mitigate wave propagation effects in InSAR and the development of algorithms and software for advanced remote sensing radar systems. Relevant projects are the Shuttle Radar Topography Mission (DLR, NASA, ASI), TerraSAR-X (DLR), Terrafirma (ESA) and the Sentinel-1 Ground Motion Monitoring Service Germany. In 2017, he was honored as "DLR Senior Scientist" for his application-oriented work within projects. 